\title{
Controlling Capillary-Driven Fluid Transport in Paper-Based Microfluidic Devices Using a Movable Valve
}

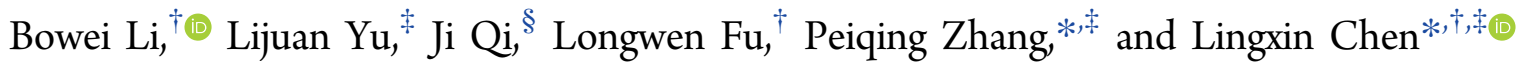 \\ ${ }^{\dagger}$ Key Laboratory of Coastal Environmental Processes and Ecological Remediation, Yantai Institute of Coastal Zone Research, Chinese \\ Academy of Sciences, Yantai 264003, China \\ ${ }^{\ddagger}$ College of Chemistry and Chemical Engineering, Yantai University, Yantai 264005, China \\ ${ }^{\S}$ School of Environment and Materials Engineering, Yantai University, Yantai 264005, China
}

Supporting Information

ABSTRACT: This paper describes a novel strategy for fabricating the movable valve on paper-based microfluidic devices to manipulate capillary-driven fluids. The movable valve fabrication is first realized using hollow rivets as the holding center to control the paper channel in different layer movement that results in the channel's connection or disconnection. The relatively simple valve fabrication procedure is robust, versatile, and compatible with microfluidic paper-based analytical devices ( $\mu$ PADs) with differing levels of complexity. It is remarkable that the movable valve can be convenient and free to control fluid without the timing setting, advantages that make it user-friendly for untrained users to carry out the complex multistep operations. For the perform-
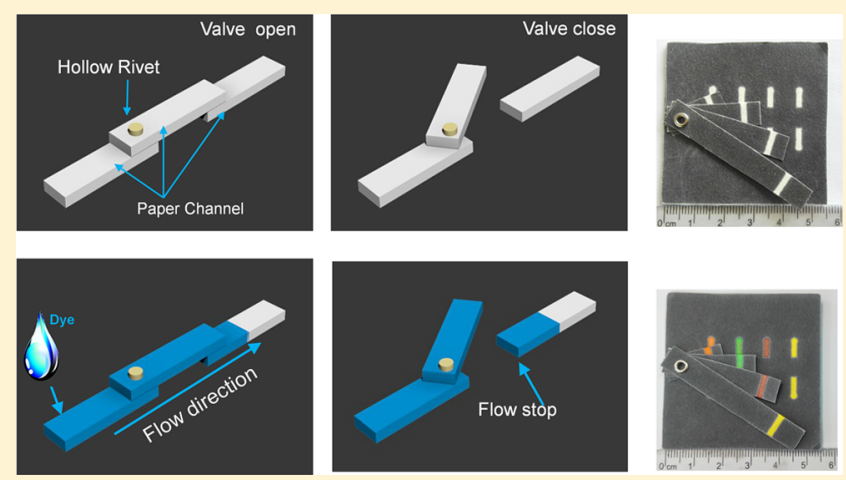
ance of the movable valve to be verified, several different designs of $\mu \mathrm{PADs}$ were tested and obtained with satisfactory results. In addition, in the proof-of-concept enzyme-linked immunosorbent assay experiments, we demonstrate the use of these valves in $\mu \mathrm{PADs}$ for the successful analysis of samples of carcino-embryonic antigen, showing good sensitivity and reproducibility. We hope this technique will open new avenues for the fabrication of paper-based valves in an easily adoptable and widely available way on $\mu$ PADs and provide potential point-of-care applications in the future.

$\mathrm{P}$ aper-based microfluidics has shown great potential over the past few decades as a promising and powerful platform. ${ }^{1-4}$ It mainly relies on attractive features including cheapness, ease-of-operation, lightweight transport, and compatibility with biological samples. Moreover, the cellulose papers can wick aqueous samples by capillary force, allowing them to operate without any external pump. Inspired by this technique, many researchers have put significant efforts toward the development of microfluidic paper-based analytical devices ( $\mu$ PADs), and this technology has been developing as quickly as a bamboo shoot after a spring rain. ${ }^{5-10}$ However, when more complicated or multistep assays are required for the $\mu \mathrm{PADs},{ }^{5,11,12}$ how the fluid should be manipulated is of great importance for realizing the essential function of performing multiple processing steps and assay detection for various applications. ${ }^{13-15}$ As a crucial and essential functional component for controlling fluid transport, the valve plays an important role in fluid manipulation and enhances the fluidic capability of $\mu$ PADs.

There is a set of techniques that was reported for manipulating fluid to realize the valve function of $\mu \mathrm{PADs}$ by relying on various mechanisms. ${ }^{16-19}$ This mainly consists of three strategies. First, the channel's geometry can be varied to result in different arrival times for controlling the liquid flow.
$\mathrm{Fu}$, Osborn, and Songok have demonstrated flow delay through changing the shape of the channel. ${ }^{20-22}$ The second strategy makes use of chemicals to influence physicochemical properties, including the viscosity of the fluid and the homogeneity of the paper to manipulate the fluid. For instance, the implementation of flow delay could be achieved by using chemical reagent such as dissolvable sugar, ${ }^{23}$ paraffin wax, ${ }^{10,24}$ surfactant reagents, ${ }^{25}$ triboelectric effect, $^{26}$ target-responsive hydrogels, ${ }^{27,28}$ and so forth. The third strategy utilizes mechanical means to control the capillary-driven fluid movement. Martinez reported multilayer stacked paper devices containing a push button valve that could be closed by mechanical compressing force. ${ }^{16} \mathrm{Fu}$ et al. utilized an absorbent sponge constructs for time-metered valves $^{18}$ and designed tunable-delay shunt valves to perform sequential delivery. ${ }^{29}$

However, these methods suffer from certain restrictions. The implementation of flow delay by a channel's geometry lacks flexibility, and most valves such as the sponge and valve can only work once because the compressed sponge would not recover when it expanded after encountering the liquid.

Received: February 27, 2017

Accepted: May 5, 2017

Published: May 5, 2017 

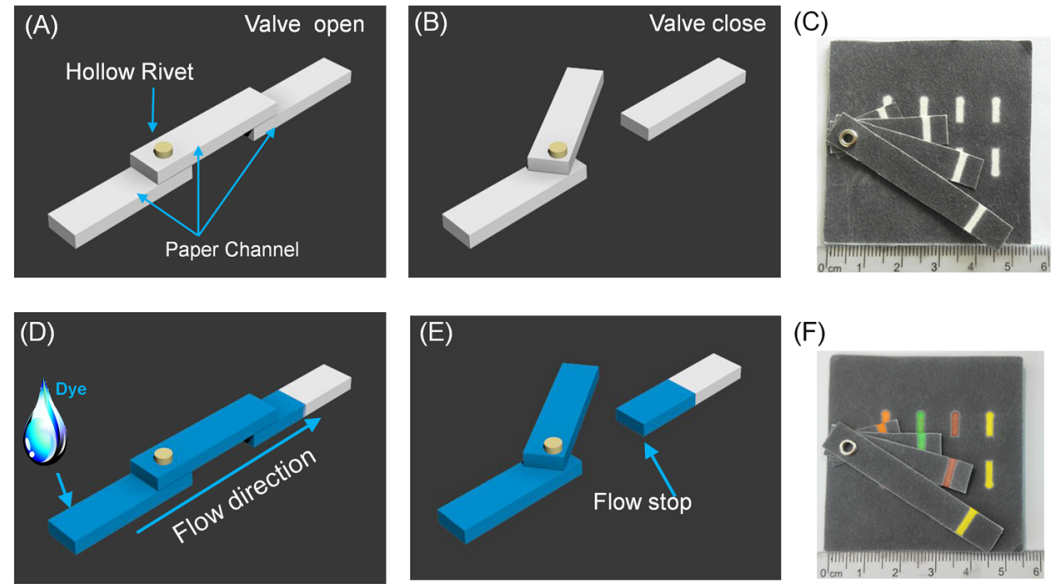

Figure 1. Schematic diagram of the hollow-rivet-assisted movable valve paper device. (A, B) Pictures showing the movable channel rotating around the pivot (yellow pillar) and causing the valve to open and close. (D) Blue dye introduced at the left end of the channel can wick through the movable channel to the right channel by capillary force. (E) The movable channel is disconnected from the right channel, stopping the flow. (C, F) Images of paper chips containing the RAM valves and loaded with different color dyes.

Moreover, the use of chemical materials may add some additional processing steps and cause inconvenience for the device. Therefore, designing a simple, flexible, and reusable valve is urgently needed for use in performing multistep or complicated assays on $\mu \mathrm{PADs}$ but is a significant challenge.

To the best of our knowledge, the present work represents the first demonstration of paper microfluidic valves using the movable valve strategy. The hollow rivet-assisted movable (RAM) valve dramatically simplifies valve fabrication processing and avoids the time delay setting (decreasing the valve's response time). In addition, because the RAM valve is easily controlled by personal demand, it can be widely suitable for various types of targets on $\mu$ PADs with different complexities. Moreover, this method can be applied in complicated multiple processing steps and assays, such as the enzyme-linked immunosorbent assay (ELISA) procedure. We demonstrated the use of these valves in $\mu$ PADs for successful ELISA analysis of the samples of carcino-embryonic antigen (CEA) that show good sensitivity and reproducibility. We believe that this technique can be used to develop new $\mu$ PADs with improved performance and features.

\section{EXPERIMENTAL SECTION}

Chemicals and Materials. All chemicals used were of analytical grade. Whatman No. 1 chromatography filter paper was purchased from GE Company (Shanghai, China). Different colors of food dyes were purchased from a local supermarket. The hollow rivets, hammer, and hole puncher were obtained from local stores. $\mathrm{HCl}, \mathrm{NaOH}$, hydroxylamine, citric acid, and sulfonamides were purchased from Sinopharm Chemical Reagent (China). CEA mouse monoclonal capture antibody, and signal HRP-labeled antibody were purchased from Linc-Bio Science Co. Ltd. (Shanghai, China). Tetrabromophenol Blue was purchased from J\&K Scientific Company. Bovine serum albumin (BSA) was obtained from Sigma. Ultrapure water purified with a Milli-Q system $\left(18 \Omega \mathrm{cm}^{-1}\right.$ resistance) was used in all aqueous solutions and rinsing procedures. The washing buffer was phosphate buffer solution (PBS) containing $0.05 \%$ Tween-20 ( $\mathrm{pH}$ 7.4). The blocking buffer was phosphate buffer solution (PBS) containing 1\% BSA ( $\mathrm{pH} 7.4)$ used for blocking the residual reactive sites on the antibody-immobilized paper.
The clinical human serum samples were obtained from Yantai Guanghua hospital.

Fabrication of the Paper-Based Chip. The paper fabrication process was the same as that in our previous reports. Briefly, we use a printer (XEROX Phaser $8560 \mathrm{DN}$ ) for designing a structure that contains hydrophobic and hydrophilic areas on the paper chip. After $30 \mathrm{~s}$ of wax melting at 150 ${ }^{\circ} \mathrm{C}$, the wax penetrated the paper, creating a barrier that blocks the flow of water.

Fabrication of Movable Valves. In this approach, a novel hollow-rivet-assisted movable valve is presented for the first time for $\mu$ PADs (shown in Figure 1). The devices could be easily assembled by hollow rivets and pins. Importantly, this movable valve method does not require adhesive tape, avoiding nonspecific adsorption and cross contamination and eliminates the need for filling of cellulose and tedious alignment. For use in assembling the paper-based microfluidic chip, hollow copper rivets, a hammer, and a hole puncher were purchased from a local shopping store. As shown in Movie S1, $4 \mathrm{~mm}$ diameter circles matching the size of the hollow rivets were located on the designed position of every layer of paper, and the $4 \mathrm{~mm}$ holes were directly drilled on these papers before the alignment; then, the hollow rivets were put through all of the pieces of paper and mounted because the rivet has a smooth cylindrical shaft on one end. When the rivet is punched by the hammer during the installation, the end is deformed, and expands to approximately 1.5 -times wider than the original shaft diameter, which allows the rivet to become fixed in this position. Fortunately, because of the soft structure of the paper, the individual paper layer can be easily rotated horizontally around the rivet, and the movable channel helps to generate the valve's "ON/OFF" function (illustrated in Figure $1 \mathrm{~A}$ and B). A hard plastic plate was used as a support substrate to strengthen the device's hardness and to avoid folding creases or deformations during the experiment (Figure S1). As shown in Movie S1, the whole fabrication process is simple, versatile, and user-friendly.

\section{RESULTS AND DISCUSSION}

Operating Fluids Using Movable Valves on $\mu$ PADs. To test the performance of the movable valve, we designed several paper-based microfluidic devices $(55 \mathrm{~mm} \times 55 \mathrm{~mm})$ with 
different levels of channel complexity. The first demonstration was carried out with a simple design as shown in Figure. 2. The
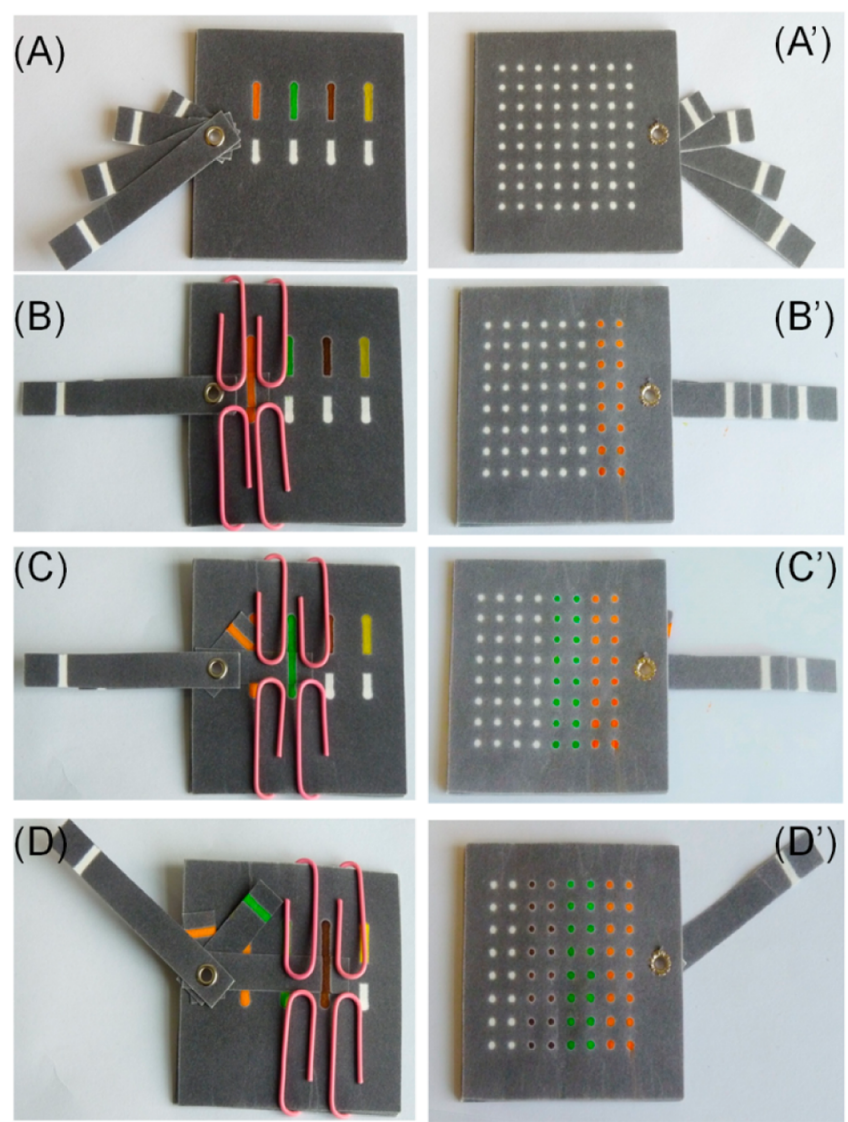

(E)
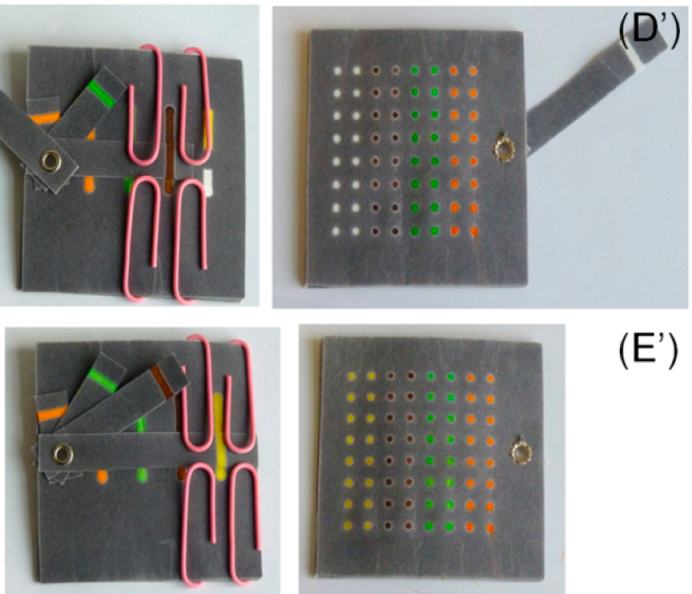

$\left(E^{\prime}\right)$

Figure 2. Top and bottom view pictures of a paper microfluidic device with dimensions of $55 \mathrm{~mm} \times 55 \mathrm{~mm}$ with four RAM valves. The black area is hydrophobic wax, and the white region is hydrophilic paper. (A, $\mathrm{A}^{\prime}$ ) Top and bottom images of the RAM- $\mu$ PADs with the valves in the "OFF" state. No color was found in the output region of the bottom. (B-E) When the four valves were in the "ON" state, four food dyes (orange, green, brown, and yellow) were wicked through the movable valves, and $\left(\mathrm{B}^{\prime}-\mathrm{E}^{\prime}\right)$ shows the locations of the 64 output region that were distributed by the dyes sequentially in four rows. An expanded view of the each layer of this device is shown in Figure S1.

chip was assembled with seven layers (Figure S2). The top layer was composed of four pieces of movable channel (like an arm) that can rotate (clockwise or counterclockwise) conveniently to connect ("ON" state) or disconnect ("OFF" state) from the second layer channel, and then the device was clamped by pins to guarantee good contact. The developed paper-based devices were capable of distributing four different samples to 64 detection regions on the bottom layer. Different colored food dyes (1:5 diluted in distilled water) were used for the visualization. As illustrated in Figure 2, when the four valves were in the "OFF" state (Figure 2A and $\mathrm{A}^{\prime}$ ), all of the channels were disconnected and fluid could not pass through to the other side of the channel; therefore, it could clearly be observed that the output reservoirs do not have any colors. In contrast, when the valves were in the "ON" state, four different dyes (orange, green, brown, and yellow) could be diverted to the movable channels sequentially; then, the dyes spread and dyed the whole channels. Furthermore, it was worth noting that if the four RAM valves were opened sequentially (shown in Figure $2 \mathrm{~B}-\mathrm{E}$ ), the food dyes could wick to the detection zones evenly. In Figure $2 \mathrm{~B}^{\prime}-\mathrm{E}^{\prime}$, when the RAM valve switched to the "ON" state, the liquid could quickly be distributed to the detection regions and did not require the response time necessary for a compressed sponge valve, which can significantly reduce the waiting time, especially for point-ofcare testing. Importantly, contingent on the rivet being a permanent mechanical fastener, this valve can work many times rather than only a single time such as for sponge ${ }^{18}$ and tunabledelay shunt valves. ${ }^{29}$ This advantage provides a good solution for complicated biological assays such as ELISA detection.

When we conceptualized this new valve fabrication procedure, we wondered if this strategy could be applied to more complicated $\mu$ PADs. Therefore, we designed two different paper-based devices containing 5 and 7 layers, respectively, to test the distribution capability (Figure S3A and B). As shown in Figure 3A and B, the paper-based devices developed were designed to distribute four samples (approximately $55 \mathrm{~mm} \times 55 \mathrm{~mm}$ disks of paper). The $\mu \mathrm{PAD}$ consisted of four RAM valves that were capable of distributing four samples into 16 detection regions $(3.5 \mathrm{~mm}$ diameter) on the bottom layer. This movable valve could work flexibly and exclude the tedious fabrication and alignment necessary for layered paper and tape valves. ${ }^{16}$ This implies that our method has the ability to integrate valves at different positions on $\mu$ PADs to satisfy the different needs of a variety of paper chips.

This new proposed method also conveniently enables valve operation at the intermediate layer of $\mu$ PADs. To further demonstrate this function, we designed a RAM valve paper chip $(55 \mathrm{~mm} \times 25 \mathrm{~mm})$ with the logo "YIC", and the movable valve layer was sandwiched between the top and bottom layers. As shown in Figure S3C, the logo "YIC" is the abbreviation of our institute (Yantai Institute of Coastal Zone Research), and the experiment revealed that fluid delivery and the corresponding lack of delivery could be carried out well through this mechanism (Figure 3C). The characteristic of using a RAM valve operating at an intermediate layer expands the diverse application of $\mu$ PADs.

However, when this new fabrication procedure was developed, there was concern that the pins would affect the reproducibility of assays. The behavior of paper wicking in multidimensional channels clamped by paper pins also complied with the Washburn equation..$^{20,23}$ A separate experiment revealed that reproducibility was minimally affected by the pins. An orange dye solution was distributed on 16 test zones, and the signal intensity was measured by the homemade paper-based colorimetric readout device ${ }^{8}$ (displayed in Figure S4). It showed a graph of the intensity of orange dye in the outlet spots, and the average value of the signal intensity was approximately 940 (Figure S5A and B). We also tested the corresponding signal of $1-4$ points using 10 different $\mu \mathrm{PADs}$, and the relative standard deviation (RSD) was $1.1 \%$ (Figure $\mathrm{S} 5 \mathrm{C}$ ). This data indicated that the changes in color intensity were nearly uniform and showed good reproducibility in our RAM devices. This result was consistent with that of other relevant experiments. ${ }^{1}$ 

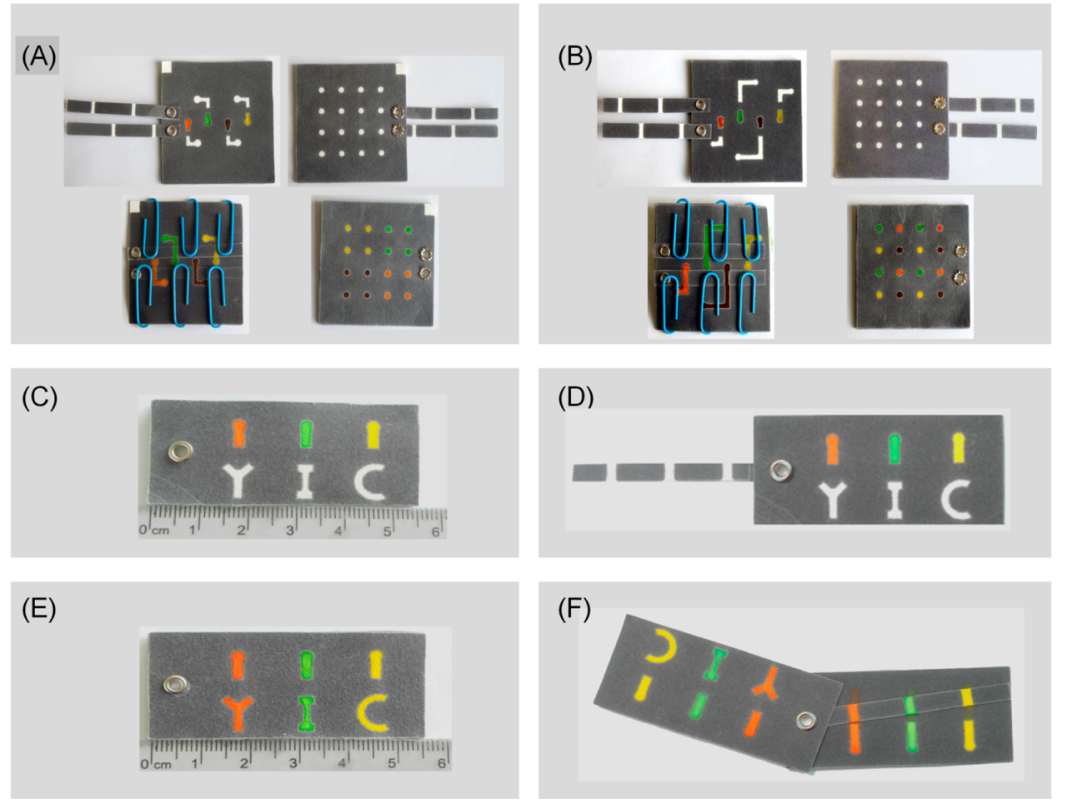

Figure 3. Design of two RAM valve paper microfluidic devices $(55 \mathrm{~mm} \times 55 \mathrm{~mm})$ that distribute four samples. (A) Top and bottom views for 5 layer $\mu$ PADs with the bottom dyed spots consisted across the four groups. (B) Top and bottom views for 7 layer $\mu$ PADs with the bottom dyed spots separated by other colors. (C-F) Photographs showing that the movable valve in the intermediate layer of $\mu$ PADs $(55 \mathrm{~mm} \times 25 \mathrm{~mm})$ functions well. (C, D) When the valve was in the "OFF" state, the YIC (Yantai Institute of Coastal Zone Research, CAS) logo was not dyed. (E, F) When the valve was in te "ON" state, the YIC logo was stained by three colors (orange, green, and yellow), respectively.

(A)
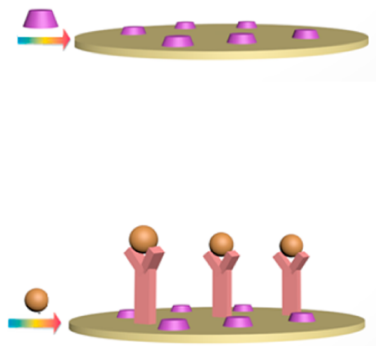

Paper
Chitosan-GA

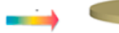

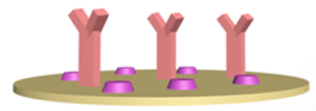

Colorless $\left(\mathrm{TMB}+\mathrm{H}_{2} \mathrm{O}_{2}\right.$ ) Blue

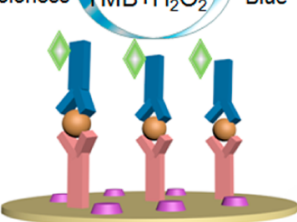

BSA

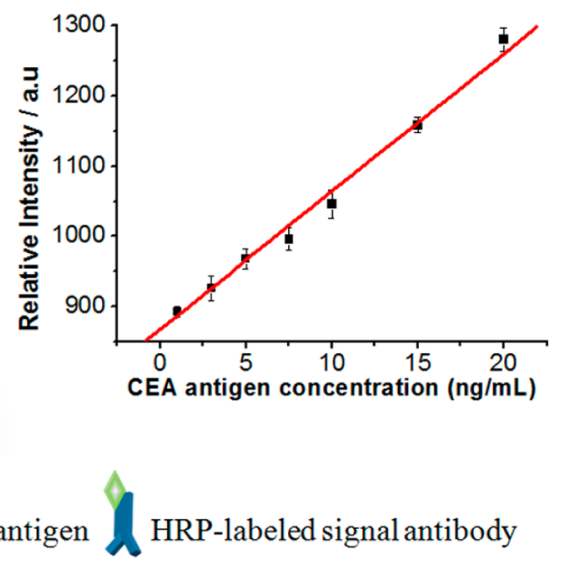

(B)

Figure 4. (A) Schematic diagram of the CEA immunoassay procedure on the RAM $\mu$ PADs. (B) Calibration curves for the determination of CEA under optimal conditions $(N=3)$.

ELISA Assay Analysis on $\mu$ PADs. ELISA has been performed on paper-based microfluidic devices by many research groups. ${ }^{30-32}$ However, the whole ELISA process is very complicated, primarily because of capture antibody immobilization, sample dispensing, and various methods for the detection of antibodies. Importantly, a washing procedure was needed during each step. Thus, controlling fluid transportation is essential for ELISA reactions. Most previous work used three-dimensional origami paper devices with folding to generate the ELISA washing process. ${ }^{14,33,34}$ To further validate the applicability of the RAM valves on paper-based microfluidic chips for bioanalysis, we carried out ELISA analysis of carcinoembryonic antigen (CEA), which is a typical biomarker for cancer diagnosis, as a proof-of-concept experiment.
The chip contained two layers and was assembled with two RAM valves, and the circle area in the middle channel was a test zone for the ELISA analysis (as displayed in the Figure S6). The main immobilization steps followed for the immunoassays were from previously described reports. ${ }^{30,31,35}$ As shown in Figure 4, a total of $5 \mu \mathrm{L}$ of $0.25 \mathrm{mg} / \mathrm{mL}$ of chitosan solution was added to each detection zone, and $5 \mu \mathrm{L}$ of $2.5 \%$ glutaraldehyde (GA) was dropped into each test zone to activate the grafting of the aldehyde group to the paper surface. After washing the ungrafted zone using $10 \mu \mathrm{L}$ of PBS washing buffer (containing $0.05 \%$ Tween-20, pH 7.4), $5 \mu \mathrm{L}$ of $1 \mu \mathrm{g} / \mathrm{mL}$ CEA antibodies was dispensed to the test area and incubated for $30 \mathrm{~min}$ at room temperature. After blocking the nonspecific site by BSA and washing three times, $5 \mu \mathrm{L}$ of different 
concentrations of CEA antigen were dropped onto each corresponding paper test area and incubated for $30 \mathrm{~min}$. Subsequently, after washing the test zone three times with a total of $30 \mu \mathrm{L}$ of washing buffer and cool drying, CEA HRPlabeled antibodies were dispensed to the test area to bind with the CEA antigen for $1 \mathrm{~h}$. Notably, the CEA antibodies and antigen samples were introduced to the test zone when the valve was in the "OFF" state. All of the washing steps were performed with the valve in the "ON" state, letting the wash buffer move through the test zone expediently and guaranteeing an effective elution. Compared to the dissolvable fluidic valve ${ }^{23}$ and origami method, ${ }^{14,31}$ this proposed strategy was easy to operate and feasible. Finally, $5 \mu \mathrm{L}$ of $\mathrm{TMB}-\mathrm{H}_{2} \mathrm{O}_{2}$ (in ELISA kits) solution was added to each detection area. The CEA antigen was arranged at increasing concentrations from 1 to 3 , $5,7.5,10,15$ and $20 \mathrm{ng} / \mathrm{mL}$ for testing (in replicates of 3 ). The calibration curve showed good linear relationships between the peak currents and the values of the analyte concentrations in the range from 1.0 to $20.0 \mathrm{ng} / \mathrm{mL}$. The linear regression equation was $Y=856+20 C_{\mathrm{CEA}}$. The error bars indicated small SDs for three repeated measurements $\left(R^{2}=0.992\right)$, and the LOD was $0.3 \mathrm{ng} / \mathrm{mL}$. The results were consistent with those of previous reports on paper-based devices. ${ }^{12,35}$ We also detected the CEA in real clinical human serum samples using our proposed method and compared them with those of the hospital chemiluminescence method (displayed in Table 1).

Table 1. CEA Assays of Real Human Serum by the Proposed and Reference Methods

CEA concentration $(\mathrm{ng} / \mathrm{mL})$

$\begin{array}{cccc}\text { clinical sample } & \text { proposed method } & \text { reference method } & \text { relative error (\%) } \\ 1 & 14.25 & 15.0 & -5.0 \\ 2 & 7.85 & 7.5 & 4.7\end{array}$

There were no obvious differences, and the relative error of the samples ranged from -5.0 to $4.7 \%$, further confirming that a movable valve could facilitate the multistep operations of CEA ELISA.

\section{CONCLUSIONS}

In this work, we have demonstrated the use of a novel hollowrivet-assisted movable valve on paper-based microfluidic devices. This hollow rivet-assisted movable valve does not require any external equipment for its activity, and this property provides a versatile, one-step fabrication, cost-effective, and easily operable method for microfluidic paper-based analytical devices. Moreover, this RAM valve can move flexibly in space, an advantage enabling its reusability with good repeatability. To evaluate the RAM valve performance, we investigated several different designs of $\mu \mathrm{PADs}$ that showed good performance. As a proof-of-concept, CEA ELISA was also investigated on this platform. The results demonstrate that movable valves can offer good opportunities for use without a complicated fabrication process for performing complex multistep operations such as point-of-care testing, on-field detection, and other biochemical assays.

\section{ASSOCIATED CONTENT}

\section{S Supporting Information}

Additional information as noted in text. This material is available free of charge via the Internet at http://pubs.acs.org/. The Supporting Information is available free of charge on the
ACS Publications website at DOI: 10.1021/acs.analchem.7b00726.

Designs of various $\mu$ PADs, device for measuring colorimetric assays, and test results (PDF)

Fabrication process of a $\mu \mathrm{PAD}$ (AVI)

\section{AUTHOR INFORMATION}

\section{Corresponding Authors}

*E-mail: zhangpqytu@126.com.

*E-mail: lxchen@yic.ac.cn.

ORCID $\odot$

Bowei Li: 0000-0001-6262-8248

Lingxin Chen: 0000-0002-3764-3515

Notes

The authors declare no competing financial interest.

\section{ACKNOWLEDGMENTS}

This work was financially supported by the Natural Science Research Foundation of China (Grants 21575159 and 21205131), the Science and Technology Development Plan of Yantai (2015ZH087), and the National Key Research and Development Program of China (Grant 2016YFC1400702).

\section{REFERENCES}

(1) Martinez, A. W.; Phillips, S. T.; Whitesides, G. M. Proc. Natl. Acad. Sci. U. S. A. 2008, 105, 19606-19611.

(2) Li, B.; Zhang, W.; Chen, L.; Lin, B. Electrophoresis 2013, 34, $2162-2168$

(3) Martinez, A. W.; Phillips, S. T.; Whitesides, G. M.; Carrilho, E. Anal. Chem. 2010, 82, 3-10.

(4) Bui, M.-P. N.; Li, C. A.; Han, K. N.; Choo, J.; Lee, E. K.; Seong, G. H. Anal. Chem. 2011, 83, 1603-1608.

(5) Liu, H.; Crooks, R. M. J. Am. Chem. Soc. 2011, 133, 1756417566.

(6) Li, B.; Zhang, Z.; Qi, J.; Zhou, N.; Qin, S.; Choo, J.; Chen, L. ACS Sensors. 2017, 2, 243-250.

(7) Yamada, K.; Henares, T. G.; Suzuki, K.; Citterio, D. Angew. Chem., Int. Ed. 2015, 54, 5294-5310.

(8) Li, B.; Fu, L.; Zhang, W.; Feng, W.; Chen, L. Electrophoresis 2014, $35,1152-1159$

(9) Xu, B.; Du, Y.; Lin, J.; Qi, M.; Shu, B.; Wen, X.; Liang, G.; Chen, B.; Liu, D. Anal. Chem. 2016, 88, 11593-11600.

(10) Lu, Y.; Shi, W.; Qin, J.; Lin, B. Anal. Chem. 2010, 82, 329-335.

(11) Li, X.; Zwanenburg, P.; Liu, X. Lab Chip 2013, 13, 2609-2614.

(12) Ge, L.; Yan, J.; Song, X.; Yan, M.; Ge, S.; Yu, J. Biomaterials 2012, 33, 1024-1031.

(13) Ding, J.; Li, B.; Chen, L.; Qin, W. Angew. Chem., Int. Ed. 2016, 55, 13033-13037.

(14) Ge, L.; Wang, S.; Song, X.; Ge, S.; Yu, J. Lab Chip 2012, 12, 3150-3158.

(15) Wang, L.; Xu, C.; Zhu, Y.; Yu, Y.; Sun, N.; Zhang, X.; Feng, K.; Qin, J. Lab Chip 2015, 15, 4283-4290.

(16) Martinez, A. W.; Phillips, S. T.; Nie, Z.; Cheng, C.-M.; Carrilho, E.; Wiley, B. J.; Whitesides, G. M. Lab Chip 2010, 10, 2499-2504.

(17) Lutz, B. R.; Trinh, P.; Ball, C.; Fu, E.; Yager, P. Lab Chip 2011, 11, 4274-4278.

(18) Toley, B. J.; Wang, J. A.; Gupta, M.; Buser, J. R.; Lafleur, L. K.; Lutz, B. R.; Fu, E.; Yager, P. Lab Chip 2015, 15, 1432-1444.

(19) Li, X.; Tian, J.; Nguyen, T.; Shen, W. Anal. Chem. 2008, 80, 9131-9134.

(20) Fu, E.; Lutz, B.; Kauffman, P.; Yager, P. Lab Chip 2010, 10, 918-920.

(21) Osborn, J. L.; Lutz, B.; Fu, E.; Kauffman, P.; Stevens, D. Y.; Yager, P. Lab Chip 2010, 10, 2659-2665.

(22) Songok, J.; Toivakka, M. Microfluid. Nanofluid. 2016, 20, 63. 
(23) Lutz, B.; Liang, T.; Fu, E.; Ramachandran, S.; Kauffman, P.; Yager, P. Lab Chip 2013, 13, 2840-2847.

(24) Noh, N.; Phillips, S. T. Anal. Chem. 2010, 82, 4181-4187.

(25) Chen, H.; Cogswell, J.; Anagnostopoulos, C.; Faghri, M. Lab Chip 2012, 12, 2909-2913.

(26) da Silva, E.; Santhiago, M.; de Souza, F. R.; Coltro, W. K. T.; Kubota, L. T. Lab Chip 2015, 15, 1651-1655.

(27) Tian, T.; Wei, X.; Jia, S.; Zhang, R.; Li, J.; Zhu, Z.; Zhang, H.; Ma, Y.; Lin, Z.; Yang, C. J. Biosens. Bioelectron. 2016, 77, 537-542.

(28) Wei, X.; Tian, T.; Jia, S.; Zhu, Z.; Ma, Y.; Sun, J.; Lin, Z.; Yang, C. J. Anal. Chem. 2015, 87, 4275-4282.

(29) Toley, B. J.; McKenzie, B.; Liang, T.; Buser, J. R.; Yager, P.; Fu, E. Anal. Chem. 2013, 85, 11545-11552.

(30) Wang, S.; Ge, L.; Song, X.; Yu, J.; Ge, S.; Huang, J.; Zeng, F. Biosens. Bioelectron. 2012, 31, 212-218.

(31) Liu, W.; Cassano, C. L.; Xu, X.; Fan, Z. H. Anal. Chem. 2013, 85, 10270-10276.

(32) Zhang, Y.; Gao, D.; Fan, J.; Nie, J.; Le, S.; Zhu, W.; Yang, J.; Li, J. Biosens. Bioelectron. 2016, 78, 538-546.

(33) Li, W.; Li, L.; Li, S.; Wang, X.; Li, M.; Wang, S.; Yu, J. Sens. Actuators, B 2013, 188, 417-424.

(34) Li, X.; Liu, X. Y. Adv. Healthcare Mater. 2016, 5, 1326-1335.

(35) Liu, W.; Guo, Y.; Zhao, M.; Li, H.; Zhang, Z. Anal. Chem. 2015, 87, 7951-7957. 